\title{
Stability Research on the Effect of Oil Spill Dispersant II - Impact of Wave Intensity
}

\author{
Qiao-min Wang, Bing Sun*, Zhi-yu Yan, Xiao-mei Zhu, Hui Liu and Yan-bin Xin
}

Environmental Science and Engineering College, Dalian Maritime University, Dalian, 116026, China

\begin{abstract}
The oil spill dispersant (OSD) play an important role to deal with the frequent oil spills in the marine environment, which can effectively reduce the interfacial tension between the oil and the seawater. However, there are many studies showing the stability of the OSD effect changes over time, and is related with the wave. In this paper, the results show the OSD will gradually move away from the oil, and the quantity and speed of the removed OSD is influenced by the intensity and duration of the wave action. The stability mechanism of the OSD effect is proposed in this study. The results of this study provide methods for the research on the stability mechanism of the OSD, as well as the theoretical basis for the comprehensive evaluation and the rational use of the OSD.
\end{abstract}

Keywords: Effect, oil spill dispersant, stability, wave action.

\section{INTRODUCTION}

As is known, the oil spill dispersant (OSD) play an important role to deal with the frequent oil spills in the marine environment, which can effectively reduce the interfacial tension between the oil and the seawater. But the effect of the OSD is not always persisted. The stability research of the dispersion effect of the oil treated with the OSD helps to grasp accurately the harm to the Marine environment, and understand the final end-result of the oil spill at sea.

The dispersion of the oil spills depends on not only the chemical properties of the oil and the OSDs, but also the changeable marine environment [1]. Breaking waves cause velocity shear and hence result in the mixing of oil and dispersant and the dispersion of oil slick [2-4]. Fingas [5] studied countermeasures of the oil spills, discovering that the most important factors are the speed of the wind and the height of the wave that are provided by the mixing energy, and the two factors are correlated with each other. When the actual operation is in the marine environment, only testing the mixing energy is not enough, which is primarily because the dilution and transmission of the seawater are not considered [6]. Concerning the physical or chemical dispersion process of the oil spills, the initial decomposition (including the second decomposed into smaller droplets) or sinking of the oil slick in the seawater depends on the turbulence, which has an important effect on the vertical transfer of the oil droplets. There are lots of researches showing waves, in particular, play a critical role in the dispersion of the oil slick by generating velocity shear to break up and transport oil in their turbulent flows [7].

Taking into account the internal and external factors described above, representative crude oil and the oil spill

\footnotetext{
*Address correspondence to this author at the Linghai Road, Dalian, China. Postcard: 116026; Tel: 0086-0411-84725275;

E-mail: sunb88@dlmu.edu.cn
}

dispersant (OSD) were selected as the experimental subjects in the primeval research. The standing experiment and dynamics experiment were investigated to study the stability and dynamic characteristics of the OSD effect. The study showed the OSD will gradually move away from the oil, and waves play a key role in the combination stability of the OSD and the oil. Weather the wave action impacts the stability of the OSD effect or not is the basis to the mechanism research. In this paper, the dynamic characteristics were studied dynamically under the continuous wave action in the different intensities, and the stability mechanism was explored as well. The analysis methods focused on the relative content of the OSD in the oil slick and the concentration of the dispersive oil in the seawater.

\section{MATERIALS AND METHODS}

\subsection{Materials}

The experimental oil was from a platform in Bohai and is medium viscosity. The OSD was the FuKen- II OSD from Qingdao Huahai Environmental Protection Industry Co., Ltd. The salinity of the artificial seawater was about $34 \%$.

\subsection{Methods}

The mixture of the oil and the OSD was poured into the seawater tank with the artificial waves. In the experiment the ratio of the OSD, oil and seawater (volume ratio), is 2:5:5500. The way the wave was generated was: the immersed part of vertical plate in the seawater was about $5 \mathrm{~cm}$ long, immersed area about $15 \mathrm{~cm} \times 5 \mathrm{~cm}$, the amplitude about $5 \mathrm{~cm}$, and the vibration frequencies were $0,33 \pm 1,69 \pm 1$ and $99 \pm 1$ times/min, respectively. All the experiments were finished at $15 \pm 1{ }^{\circ} \mathrm{C}$ in the laboratory. Each experiment had been maintained for 12 hours, the relative contents of the OSD in the oil slick and the concentration of the dispersive oil in the water were measured per hour during the experiment. The location of seawater sampling was $10-13 \mathrm{~cm}$ away from the surface. 


\subsection{Test Methods}

\subsubsection{The Relative Content of the OSD in the Oil Slick}

The relative content of the OSD in the oil slick was measured by the gas chromatography (Shimadzu Co., Ltd. GC-2010 gas chromatography). The pretreatment of the oil slick and the testing conditions of the GC are identical to the stability research of the Part-I.

\subsubsection{The Concentration of the Dispersive Oil in the Sea- water}

The concentration of the dispersive oil in the seawater was measured by the Ultraviolet spectrophotometer (T6-UV spectrophotometer of Purkinje General).

The seawater sample was taken with $20 \mathrm{~mL}$, which was successively extracted by $20 \mathrm{~mL}$ and $10 \mathrm{~mL}$ mixture of dichloromethane and n-hexane (the volume ratio is $2: 1$ ) several times, and then merge the extract liquor. The extract liquor was leached by chromatographic column described above, collected and sealed for testing.

\section{RESULTS}

The study about the OSD's stability is aimed at exploring the dynamic characteristics of the dispersive oil and the temporal characteristics of the OSD's stability, when the oil spill is operated by the continuing and different intensities of artificial wave action. Test content in this study is divided into two parts. One is the oil slick analyzed by gas chromatography, which is used to analyze the loss of the OSD; the other is the concentration of oil in the seawater analyzed by UV spectrophotometer, which is used to analyze the change of the ability of dispersion.

\subsection{The Change of the Relative Content of the OSD in the Oil Slick}

The GC results of the relative content of the OSD in the oil slick are shown in Fig. (1).

As is shown in Fig. (1), the general decreasing trend is far less than that in the Fig. (1), which manifests the OSD in the oil slick slowly move away from the oil slick, but the waves play an important role to keep the OSD in the oil for a long time.

The obvious decreasing curve in the Fig. (1) presents fluctuation characteristics, which shows the OSD had dynamic and reversible mass transfer between the oil slick and the seawater. The oil added into the OSD was largely dispersing when poured into the seawater, but the OSD gathered in the oil-water surface due to the thermal motion, thereby the redundant OSD dispersing into the interstitial seawater; while some dispersive oil will float to the oil slick under the effect of the buoyancy, entraining the seawater that contains the OSD. The OSD in the seawater was also pretreated by chromatography analysis, which will be extracted. As a result, the relative content of the OSD was larger than the initial's. If the entrained seawater was leaving the oil slick, it was not sampled and analyzed due to its instability, which made the relative content of the OSD in the oil slick decrease. This process occurs repeatedly, causing the fluc- tuations in falling of the relative content of the OSD, rather than diminishing directly.

The amount of the mass transfer is reflected by the fluctuation amplitude. As can be seen from the Fig. (1), the fluctuation amplitude is relevant to the frequency of the wave (the intensity of the wave). When the frequencies of the wave are $0,33 \pm 1,69 \pm 1,99 \pm 1$ times/min, respectively, the variation ranges of the relative content of the OSD are about $0.3-0.48,0.3-0.55,0.34-0.52$ and $0.31-0.47$, respectively, which shows that with the wave velocity increasing, the variation range of the relative content of the OSD is smaller. The reason above shows that the more adequate mixing energy from the waves is, the harder OSD is to be away from the oil, and that the enough mixing energy can help the OSD remain in the oil.

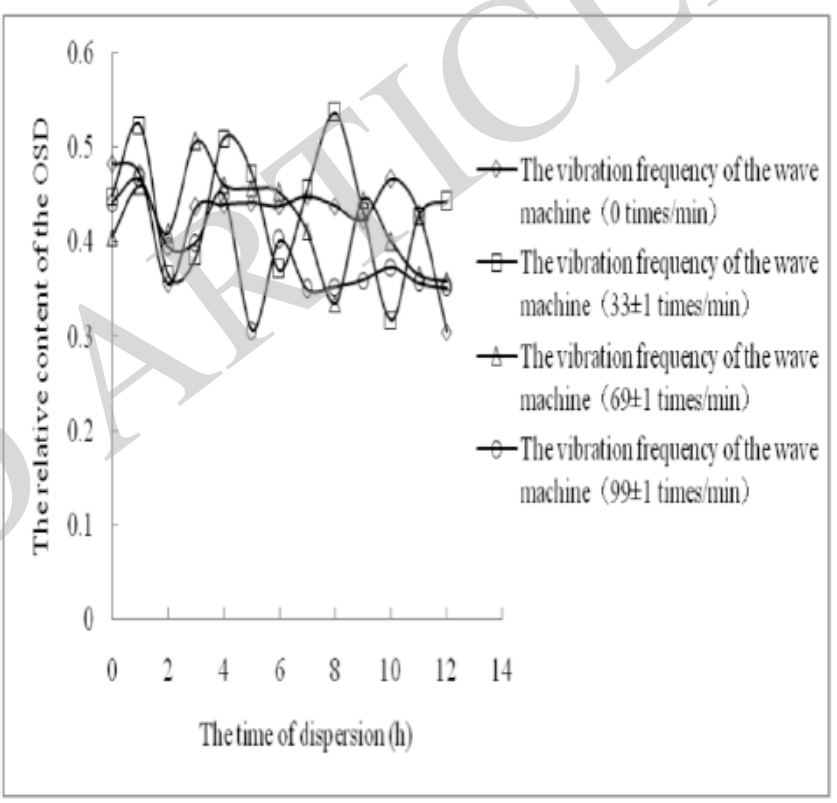

Fig. (1). The change of the relative content of the OSD in the oil slick with the time in the dynamic experiment.

When the wave action time is below $4 \mathrm{~h}$, the relative content of the OSD is decreased by the similar fluctuation frequency. The curve is falling between $0-1 \mathrm{~h}$, rising between 1 $2 \mathrm{~h}$ and falling between $3-4 \mathrm{~h}$. Although the falling-rising frequency is fast, the changing process of the OSD leaving from the oil slick is slow. The fluctuations of the four curves are no longer similar after $4 \mathrm{~h}$, which could be related with the wave frequencies and the long-time combined effect of the reversible process (oil droplets' dispersion and aggregation, the OSD's attachment and detachment in the oil-water interface, and the entrainment and separation of the seawater).

The conclusions are obtained from the above analysis: the OSD can be away from the oil slick; the OSD can gradually move away the oil slick during the reversible process (the dispersion and aggregation of the oil, the entrainment and adherence of the seawater, and the OSD's adhesion and separation); the wave contributes to the combination stability of the oil and the OSD. 


\subsection{The Change of the Oil Concentration in the Seawater}

The change of the dispersive oil concentration of the seawater is measured by the UV spectrophotometer. The results are shown in Fig. (2).

As is shown in Fig. (2), the dispersive oil concentration of the seawater shows a certain degree volatility with the different wave frequencies, mainly because the oil in the seawater exist under the two states of motion, which constantly disperses and goes up. Besides, the sample is collected at a certain point in a certain depth of the seawater, and the rising and falling speed of the oil droplets here is not the same so that the concentration will fluctuate. When the frequency of the wave is fast, the process of the dispersion and the floating will be increased, which make the volatility of the oil concentration increase; but if the speed of the wave is excessively fast or the action time is increased, the degree of the mixing will be also increased in the limited tank, and the fluctuation characteristics of the oil concentration are irregular. Furthermore, the oil concentration increases with the increasing of wave frequency, which indicates the strong waves can help more dispersive oil remain into the seawater.

In summary, the testing result of the oil concentration in the seawater is the same as the testing result of the OSD, which also reflects the process of the dispersion and the floating.

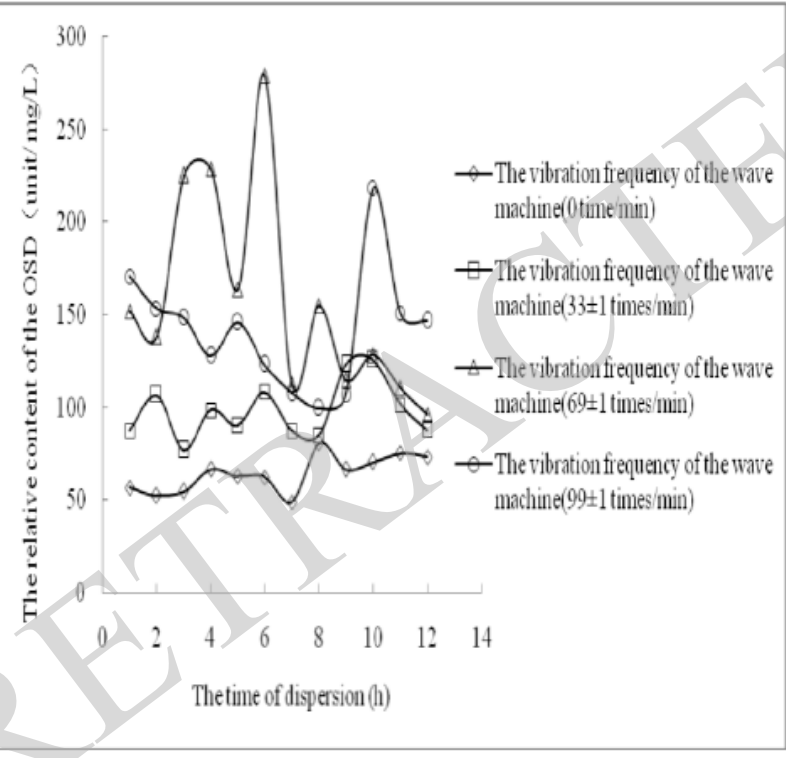

Fig. (2). The change of the dispersive oil concentration of the seawater with the time in the dynamic experiment.

\section{DISCUSSION}

Through the analysis and discussion of the experimental results of the Part 3, the transfer mechanism of the OSD at the oil-water interface can be obtained preliminarily.

When the mixture that is thoroughly mixed is dumped into the seawater, the mixture is mixed with the seawater by the gravity and the OSD would diffuse into the seawater by the concentration difference. Due to the reason above, the OSD has a directional movement towards the interface of the oil and the seawater, which makes the oil disperse into the seawater. The oil and the seawater will be further mixed under the wave action, but because the density of the mixture was less than seawater, it will go up to the surface. The oil slick in a limited tank will gather and entrained seawater to form stable oil-in-water emulsion, which will be dispersive and entrained seawater again under the action of the waves. Part of the OSD can be away from the oil slick in the reversible process of the oil's dispersion, floating and gathering, and the seawater's entrainment and separation. Beyond that, there are also some of the OSD in the oil-water interface entering the seawater, and then will be entrained into the oil slick. But due to the instability of the entrained seawater, it will be separated from the oil slick, and then get into the seawater. It is possible to be entrained if the OSD does not stay away from the oil slick, so repeatedly. This process above repeated quickly and continuously under the waves action, so that the OSD in the oil slick indistinctively declines in the overall trend, but the speed of the OSD getting into the seawater is faster than that of the OSD entrained back to the oil slick, and ultimately the OSD move away from the oil slick getting into the seawater. The specific process is shown in Fig. (3). Thus it can be seen that wave plays a key role in dispersion stability of the OSD, but different intensities of the wave will give the system different energy. Therefore, although the mixture has the same movement tendency, the characteristics presented with time is different.

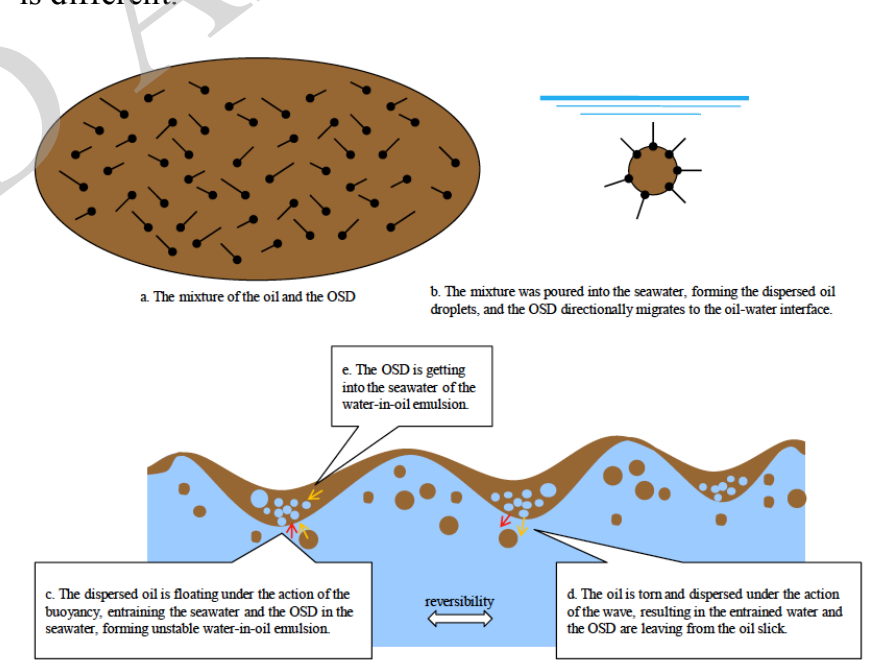

Fig. (3). The oil spill dispersant action mechanism in the schematic diagram.

The simulation experiment in the laboratory is significantly different from the actual situation of the ocean. Due to the fact that the waves are not strong enough, the dispersion effect of the oil is not good. Most of the oil is still floating on the surface of the seawater, which also shows that the emulsion of the OSD measured in the laboratory can't represent the actual dispersion. When the dispersive oil floats to the surface, it is gathered together due to the limitations of the experimental tank, which is different from the actual oil spill in the ocean, so the dynamic characteristics of the process that the OSD is out of the oil slick are obtained under the condition of the experiment, although the OSD is out of the oil slick has been confirmed. But it can be deduced that if the sea is calm and the broken wave don't reach the seawater, 
the OSD will be out of the dispersive oil in the actual sea state, which make the oil float on the sea, or even reaggregate.

\section{CONCLUSION}

The dynamics characteristic of the OSD was investigated at different wave's conditions in this study. The conclusions are listed below.

The changes of the OSD in the oil slick were analyzed by the gas chromatography, which manifested the OSD will move away from the oil slick whatever the conditions are.

Waves play a key role in the combination stability of the OSD and the oil, which mainly reflects the wave intensity. The intensity of the waves is more severe. The OSD is harder to separate from the oil slick.

The test result of the concentration of the oil in the seawater manifests fluctuation characteristics, which shows that the OSD moving away from the oil slick is gradually emerging into the seawater with entrained seawater during the process that the oil droplets repeatedly disperse and float.

The mechanism obtained in the experiment can infer the OSD will move away from the oil slick in the actual sea conditions, which may make the dispersive oil floats again and eventually aggregates.

\section{CONFLICT OF INTEREST}

The authors confirm that this article content has no conflict of interest.

\section{ACKNOWLEDGEMENTS}

The work was supported by the National Natural Science Foundation of China (41206095), Ph.D. Programs Foundation of Ministry of Education of China (20122125120010), and the Fundamental Research Funds for the Central Universities (No. 3132013091). The work was also supported by the project "Research on the characteristics, drifting prediction and recovery technology of the Bohai submerged and sunken oil". The authors wish to thank the Hebei Maritime Safety Administration of China for their help.

\section{REFERENCES}

[1] M.F. Fingas, Use of Surfactants for Environmental Applications. Surfactants: Fundamentals and Applications to the Petroleum Industry, In: L.L. Schramm, Ed., Cambridge University Press, 2000, pp. 461-539.

[2] J. Nilson, A. Naess, and Z. Volent, Measurements of Oil Concentrations in the Water Column Under Breaking Waves, Report STF 60 A 85079, Norwegian Hydrotechnical Laboratory, Trondheim, Norway, 1985.

[3] J.M. Shaw, "A microscopic view of oil slick break-up and emulsion formation in breaking waves", Spill Science \& Technology Bulletin, vol. 8, pp. 491-501, May-June 2003.

[4] P. Tkalich, and E.S. Chan, "Vertical mixing of oil droplets by breaking waves", Marine Pollution Bulletin, vol. 44, pp. 12191229, Nov 2002

[5] M.F. Fingas, "Dispersants: a review of effectiveness measures and laboratory physical studies", In: Proceedings of Alaska RRT Dispersant Workshop, U.S. Minerals Management Service: Anchorage, Alaska, 1991.

[6] National Research Council (NRC), Understanding Oil Spill Dispersants: Efficacy and Effects, The National Academies Press: Washington, DC, 2005.

[7] M. Li, and C. Garrett, "The relationship between oil droplet size and upper ocean turbulence", Marine Pollution Bulletin, vol. 36, pp 961-970. Dec 1998.

Received: December 15, 2014

This is an open access article licensed under the terms of the Creative Commons Attribution Non-Commercial License (http://creativecommons.org/licenses/by-nc/3.0/) which permits unrestricted, non-commercial use, distribution and reproduction in any medium, provided the work is properly cited. 